\title{
Main Phenotype Subphases in Reprogramming Somatic Cells as a Model of Cellular Differentiation Process
}

\author{
Victor Valdes pino ${ }^{1, *}$, Patricia M Valdespino ${ }^{2}$, Victor Valdes pino Junior ${ }^{3}$ \\ ${ }^{1}$ Health Attention Department, Universidad Autónoma Metropolitana, México \\ ${ }^{2}$ Institute of Ecology. Universidad Nacional Autónoma de México, México \\ ${ }^{3}$ Medical Ambulatory Attention Unit. Instituto Mexicano del Seguro Social, México \\ *Corresponding author: vvaldespinog@y ahoo.com.mx \\ Received January 25, 2013; Revised May 25, 2013; Accepted May 26, 2013
}

\begin{abstract}
The cellular differentiation process involves complex genetic, epigenetic and signaling pathways systems. The analysis of a specific model of cellular differentiation may contribute to understand the global mechanisms. The cellular differentiation process based on the experimental reprogramming of somatic cells (terminally differentiated cells) to induced pluripotent stem cells (iPSCs) can be used a study model of cellular differentiation. The cellular differentiation process includes constitutive changes in DNA damage response, chromatin remodeling, nuclear receptors, cell cycle regulation, apoptosis induction, cell adhesion and motility changes, immune recognition, metabolism routes, intercellular communication and in response to environment signals. It also includes the acquisition of changes into specialized cell subphenotypes as changes of shape, overproduction of organelles, suborganelles, control position of the mitotic spindle, preferential-transit signaling pathways and production of biomolecules with specialized functions. Different temporo-spatial genetic/epigenetic gene expression pattems and translational and posttranslational processes have been shown in the reprogramming of somatic cells. We analyze the main phenotype changes from fibroblast to iPSC (in cell cycle and cell adhesion/motility) to come after reprogramming, and use these changes as a model of cellular differentiation process.
\end{abstract}

Keywords: cellular differentiation model, reprogramming, induced pluripotent stem cells, phen otype subphase

\section{Introduction}

The cellular differentiation is a complex process that most living cells fulfill. Within de main cellular functions, the differentiation process is the less known subcellular and molecular mechanism. In the last decade, new knowledge has been unveiled about differentiation cell programs.

For mammalian cells, the decision to exit or enter in the differentiated mode is based on environmental extra cellular signals and cell-cell contact, as well as internal differentiation programs. Differentiation cellu lar programs are tightly related to asymmetrical cell division, cell-cycle exit, and to a resting state. Intracellu lar signaling cascades upregulate specific transcription genes that promote differentiation, and antagonize prolife ration.

Cellular differentiation process is practically universal among the living organisms, the use of simple models has been required to begin its understanding. Embryonic stem cells and progenitor cells differentiation have been the main cellular models to understand the mechanisms regulating cell differentiation. Other models have been used to accomplish this goal, particu larly two cell models: somatic stem cells differentiation in development and somatic cells transdifferentiation.

Genetic model system Drosophila using stem cell-like neuroblasts which generate the brain, is probably, the best known eukaryotic differentiation model of stem (neural) cell biology. Mammalian transdifferentiation model in mediator cells of innate and adaptive immune response, particularly dendritic cells and CD8+ $\mathrm{T}$ cells differentiation is one of the best known in humans. Recently, the human induced pluripotent stem cells model has revealed a great deal of genetic/epigenetic clues of the cellu lar differentiation process.

In this minireview, we highlight the current understanding of different steps and subphases of the cellular differentiation process using the somatic cells reprogramming model.

\section{Human Induced Pluripotent Stem Cells Model to Understand the Cell Differentiation Process}

Tissue homeostasis and repair upon injury depend on the regenerative capacity of distinct tissue-specific adult stem cell populations, as well as on renewal and differentiation process. Numerous intrinsic signals as well as micro-environmental cues from their niche allow stem cells to maintain genetic and epigenetic marks enabling their self-renewal [1].

The two classic major strategies to direct the fate of cell types are directed differentiation (in which cultured pluripotent stem cells are coaxed through a series of steps 
that are usually designed to mimic those that produce the desired cell type in vivo), and reprogramming or transdifferentiation. In reprogramming strategy, one fully differentiated cell type can be converted directly in vitro into another without a multipotent or pluripotent intermediate. More recently, the find ing that adult somatic cells can be reprogrammed to a pluripotent state demonstrated that drastic alterations in cell fate could be achieved with a combination of "reprogramming factors" [2]. During the conversion of somatic cells into pluripotent stem cells, the cells undergo dramatic epigenetic reprogramming and have the potential to differentiate into all cell types of a mammalian organis $\mathrm{m}$, including germ cells (under defined culture conditions in vitro) $[3,4]$.

One recent experimental model to understand the cellular differentiation process is based on the reprogramming of somatic cells (terminally differentiated cells) to the induced pluripotent stem cells, by ectopic expression of defined transcription factors. In 2006, Takahashi and Yamanaka published their milestone strategy to reprogram somatic mammalian cells to induced pluripotent stem cells (iPSCs) by over-expression of only four transcription factors, OCT4, SOX2, KLF4 and MYC (OSKM) [5,6]. These transcription factors are powerful modulators of pluripotent states and are preferentially expressed and play essential roles in both early embryonic development and maintenance of embryonic stem cells. Many studies have demonstrated that mouse and human iPSCs are morphologically, functionally and molecularly highly similar to their respective embryo-derived embryonic stem cells (ESCs) counterparts at the level of transcription and genome-wide distribution of chromatin modifications. Therefore, these transcription factors erased the somatic programme and established the pluripotent capabilities. The iPSC technology has opened the amazing prospect of autologous regenerative medic ine, and has revolutionized the fields of development biology and aging [3].

Despite the development of the use of OSKM-method, OSKM can be substituted by other factors under certain circumstances, which thus replace the OSKM functions in the conversion of differentiated cells into iPSCs [7]. However, by using any of the methods, only a small percentage iPSCs are obtained. It is now believed that the low efficiency of reprogramming is attributable to epigenetic memory and tumor suppressor gene hurdles. Extrinsic signals can modulate reprogramming as various others combinations of transcription factors and small molecules (epigenetic drugs) in relation to the transcriptional network of pluripotent cells $[8,9]$.

While reprogramming somatic cells to iPSCs with OSKM in culture dish, only few of somatic cells convert to the pluripotent state within 1 to 2 weeks, even when most of the cells are induced to express all the reprogramming factors-OSKM. The nu mber of faithfully reprogrammed colonies remains low relative to the number of divid ing cells $(3-5 \%)$ in the culture dish (Figure 1). Probably the degree of differentiation of somatic cells in a lineage influences the efficiency and kinetics of the process [10].

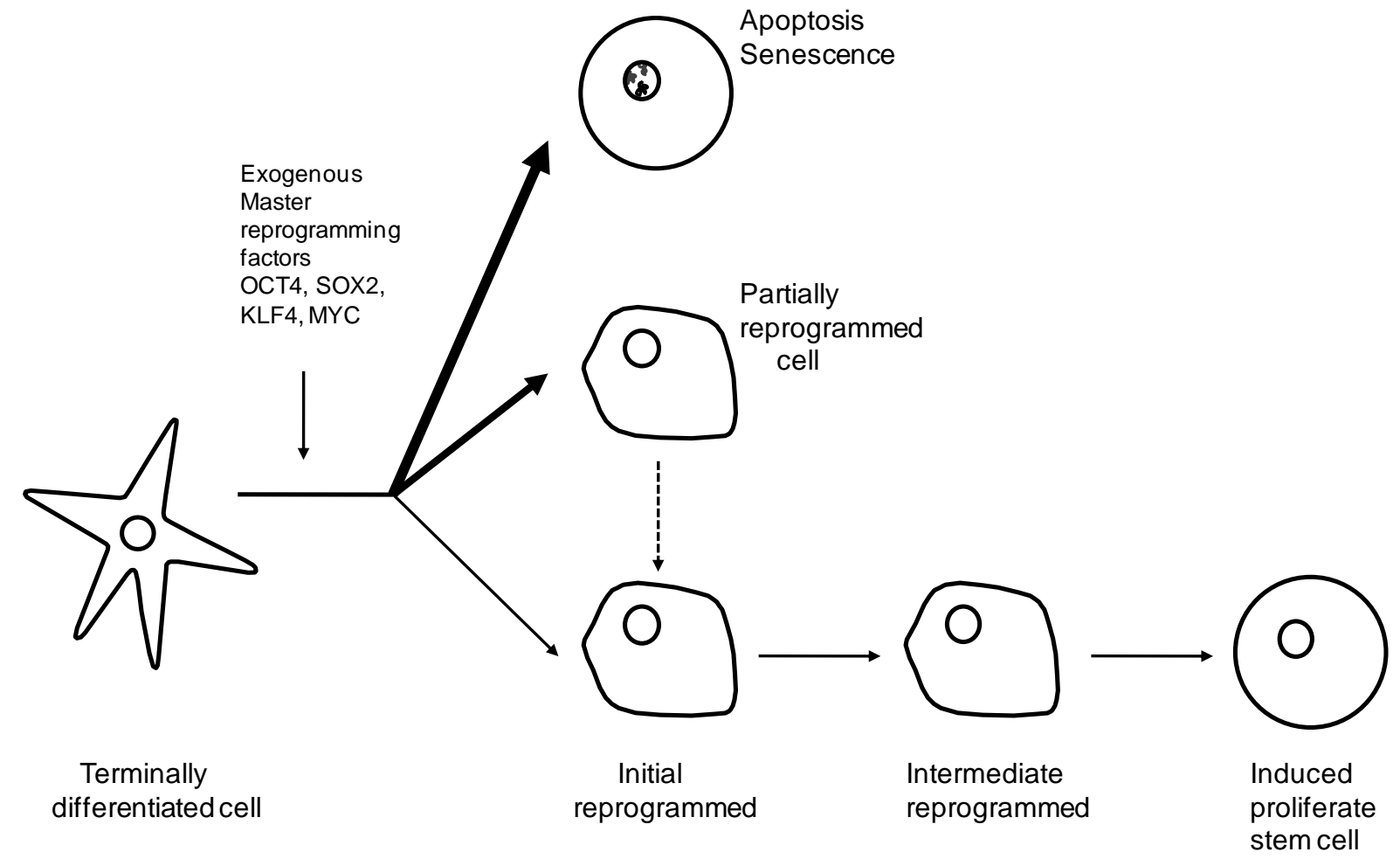

Figure 1. Reprogramming from somatic cells to induced pluripotent stem cells, by means of Takahashi and Yamanaka system [6,7]

Different studies in reprogramming have let expand our knowledge of how transcriptional networks and epigenetic modifications affect cell fate [11]. Vast quantities of data about reprogramming to pluripotency studies using "omics" technologies have been the documentary relational database of the cellular differentiation cellu lar steps. Particularly, the use of various tetracyclineinducible expression systems and live imaging analyses, has had a huge impact on mechanistic studies $[8,12]$. Normal or altered transcriptional regulation in higher eukaryotes drives to physiological programming or reprogramming process. Several mechanisms fall under 
the banner of the epigenetic machinery, changes in processes such as DNA methylation, histone modifications, and microRNA expression. Their alterations lead to temporal or permanent changes in the expression of genes that regulate the cell phenotype, such as cellular growth and cellular adhesion [13]. The generation of iPSCs is slow, with failure of a full and uniform process and have the risk of teratoma formation, e.g. when immunodeficient mice were in jected with iPSCs, some of them had well-formed teratomas [10].

Not only genetic information but also epigenetic information is replicated through each cell cycle, and each genomic organization regions is replicated at a specific time in $\mathrm{S}$ phase. Our mechanistic understanding of epigenetic processes leading to cell fate changes is still limited, but the breathtaking speed of new discoveries in the field of reprogramming will surely fill this gap. The transcription factor-induced reprogramming to the iPSCs state erases the somatic program and established the ESClike transcriptional network to confer pluripotent capabilities. It is now believed that the low efficiency of reprogramming is attributable to epigenetics hurdles (repressive chromatin states) that are only overcome infrequently.

Cell fates during development or reprogramming processes are neither restrictive nor irreversible. Distinct states of pluripotency or unipotency can interconvert through the modulation of both cell-intrinsic and exogenous factors. Various extrinsic signals can modulate reprogramming and even affect the activity of the reprogramming factors, demonstrating the close relationship of extrinsic and intrinsic pathways in regulating reprogramming and cell identity $[8,14,15]$. The iPSCs obtained by reprogramming show a wide variety of molecular changes with partial reprogrammed cells, and cells that are close to their final destination (reprogrammed state).

Mechanisms underlying epigenetic regulation play a critical role in the cellular differentiation. Epigenetic modifications regulate gene expression and define cellular identity. These modifications form the basis of cellular differentiation, through imposing memories upon the cells that determine both lineage specification and potential for production of specific cell types. Reprogramming can be considered to mimic the "resetting" that occurs during meiosis and fertilization, which allows the formation of a new individual from two older individuals [16,17]. Reprogramming field has demonstrated that differentiation is not a determin istic dead-end process but one that can be reverted experimentally. Reprogramming has been considered a central methodology for the understanding of cell $d$ ifferentiation/specialization, developmental processes, aging and aging-related disorders $[3,18,19]$.

\section{Subphenotypes Changes During the Cell Differentiation Process}

Stem cells are characterized by self-renewal and the capacity for differentiation. Pluripotent cells have the ability to form all somatic lineages, and the first pluripotent cell lines were derived from a germline tumor (teratocarcinoma). The generation of lines of embryonic stem cells from human embryos came less than a decade later, but more recently the generation of induced pluripotent stem cells has been successful. Stem cells or iPSC have biological dynamical-systems such as oscillatory gene expression; expression levels can describe the transition state between stem and differentiated cells [20].

The maintenance of tissue specific stem cell involves an exquisite balance between microenvironmental cues and cell autonomous signals. Indeed, the integration of extrinsic (e.g. leukemia inhibitory factor-LIF, bone morphogenetic protein 4-BMP4) and intrinsic signals is required to preserve self-renewal and tissue regenerative capacity of adult stem cells [10, 21]. The LIF signaling pathways leads to phosphorylation of STAT3, and BMP4 induced the expression of Id genes to suppress differentiation. Human ESCs (hESCs) require the fibroblast growth factor 2 and activin/nodal signaling pathways for proliferation maintenance [22].

The cellular differentiation process includes constitutive changes in DNA damage response [23], chromatin remodeling [24], nuclear receptors [25], cell cycle regulation [26], apoptosis induction [23,27], cell adhesion and motility changes [28,29], immune recognition, metabolis $m$ routes [27], intercellular communication, and in response to environment signal (e.g. chemosensitivity) [15]. It also includes the acquisition of changes into specialized cell subphenotypes as changes of shape, overproduction of organelles and suborganelles, control position of the mitotic spindle [30], preferential-transit signaling pathways and production of biomolecules with specialized functions [1,8]. Different genetic and epigenetic regulation of gene expression patterns takes place at several different levels of the transcriptional, translational and posttranslational processes. Both iPSCs and ESCs showed a tendency for gains at chromosomes 12 and 17, whereas iPSCs had additional gains at chromosomes 1 and 9, and ESCs at chromosomes 3 and 20. The number and size of these copy number variants (CNVs) were negatively correlated with the passage number in iPSCs. A comparison of iPSCs and their parental cell of origin showed that the majority of CNVs were created de novo in fragile regions of the genome [31,32]. Reprogramming was associated with deletions in tumor-suppressor genes, whereas extended time in culture led to duplications of oncogenes in iPSCs. Reprogramming cells to a pluripotent state entails global epigenetic remodeling and introduces epigenetic changes that are necessary for reprogramming to occur, e.g., this occurs at differentially methylated regions (DMRs). High incidence of DMRs in iPSCs compared with progenitor somatic cells suggests that these patterns are stochastic and arise during reprogramming [33].

Cell proliferation and differentiation are tightly coupled processes. In general, growth and differentiation are mutually exclusive, but they are cooperatively participating during the course of development. The process of a cell transition from $\mathrm{g}$ rowth to differentiation is of general importance for the development of an organism. When most cells are terminally differentiated, they exit the cell cycle, and cells cease division and enter to G0-phase [34]. 
As we mentioned in the cellular differentiation process, cells change the expression pattern of cellular adhesion molecules that modify the intercellular communication. Numerous cellular events that trigger differentiation, growth, cell cycle and many signaling pathways directly depend on this intercellular communication. Cells have different ways to communicate, including soluble factors like cytokines or growth factors and their corresponding membrane receptors or cell-cell adhesions. There are constitutive cell-cell connections, which are important for the development of cell polarity and the maintenance of tissue organization like cadherin connections in epithelial cells. If normal cells lose their constitutive cell-cell connections or cell-matrix connections, they go into apoptosis. The catenins ( $\alpha$ and $\beta$ ) are among the most essential cytoplasmic proteins that interact directly with the cadherins. The classical cadherins E, N, and VE are transmembrane proteins with a specific extracellular domain that bind to cadherins on other cells. The intracellular domain interacts with numerous cytoplasmic proteins, which in turn interact with the cytoskeleton and signaling pathways that can produce changes in cell motility, migration, proliferation and shape. The loss of Ecadherin is an essential event in the context of epithelialmesenchymal transition (EMT), that which regulates tissue morphogenesis and the conversion of cells that exhibit an epithelial phenotype to the mesenchymal phenotype. EMT is a process characterized by loss of cell adhesion, repression of E-cadherin expression, and increased cell motility. This condition is considered to be essential for cell epithelial dedifferentiation, and is a very critical step in cancer progression since it enables hematogenic and lymphogenic cancer cell dissemination. Other adhesion molecules that participate on tissue organization are the family of integrins and receptors that belong to the immunoglobulin gene superfamily, and are involved in the attachment of cells to the extracellular matrix and to each other [35]. Alterations of cellular adhesiveness are caused by either genetic mutations or alterations of the epigenome. Two repressors of Ecadherin expression are the transcription factors snail homolog 1 (SNAIL1) and zing finger E-box binding homeobox 1. In some types of cancer as gastric cancer, colorectal cancer and leukemia, the DNAhypermethylation even appears to be the leading mechanis m of E-cadherin suppression [35].

Genome wide epigenetic reprogramming is crucial for deriving fully reprogrammed cells. Epigenetic modulations during somatic cell dedifferentiation can overcome reprogramming barriers. Transcriptional effects induced by the OSKM reprogramming factors generate populations of partially reprogrammed cells (pre-iPSCs), that are characterized by down-regulation of somatic genes, incomplete reactivation of pluripotent genes, maintenance of viral transgene expression, inability to form chimeras, and persistent DNA hypermethylation. Preliminary results showed that in this step of cellular reprogramming some epigenetic marks may elicit various cellu lar responses, including cell proliferation, growth and death, as well as the promotion of tumorigenesis.

There is a complex network of signaling pathways in ESCs/iPSCs that can respond to extracellular stimuli that strictly control the equilibrium between self-renewal and differentiation. Different cells undergo cell-fate determination through stepwise activation of multiple signaling pathways, including the Notch, Hedgehog, Wingless (Wnt), and Janus kinase/STAT (JNK). In addition, changes in DNA replication and cellu lar growth depend on the spatial and temporal activation of the mitotic cycle-endocycle and gene-endocycle amplification cell-cycle switches [36]. Recent evidence shows that modulation of the Wnt/ $\beta$-catenin, MAPK/ERG, TGF $-\beta$ or $\mathrm{PI} 3 \mathrm{~K} / \mathrm{Akt}$ signaling pathways strikingly enhance somatic cell reprogramming. For example, WNT signaling upregulates c-Myc expression and promotes naïve pluripotency. The canonical Wnt/ $\beta$-catenin pathways represent a key regulator of stem cells self-renewal (neuronal progenitors), and inhibit the keratinocytes differentiation. The role of Notch signaling in cell proliferation and differentiation is highly cell-specific, some Notch's signaling components (LGN, NuMA, dynactin) control asymmetric cell division by reorienting mitotic spindles to achieve perpendicular divisions [30,37]. Therefore, the generation of iPSCs obtained from dedifferentiation of somatic cells takes a long path of several signaling networks.

Transcriptional effects induced by the OSKM reprogramming factors have been detected in transduced adult dermal fibroblast as early as day 4 after infection. However, only a few of the cells become stable and reactivated endogenous pluripotency marks (e.g. NANOG expression). This indicates that the majority of the infected cells were trapped in a partially reprogrammed state due to their inability to overcome some reprogramming barriers (Figure 1).

On the other hand, cellular transdifferentiation takes a shortcut to new lineages without pluripotency intermediates. Efficient conversions of somatic cell types into different somatic cell types, without going through the pluripotent state, have been achieved by overexpressing specific sets of lineage-specific transcription factors in combination with appropriate culture conditions. Experimental transdifferentiation was demonstrated from fibroblast to myoblast (overexpression of MYoD), to card iomyocites (overexpression of Gata4, MEF2c and $\mathrm{Tb} \times 5)$, to immature neuron-like cell (overe xpression of Brn2, Mytl11, Zic1, Olig2 and Ascl1), and to hematopoietic progenitors (overexpression of OCT4 and modification of culturing protocols), from B cells to macrophages (overexpression of $\mathrm{C} / \mathrm{EBP}$ ) and from adult pancreatic exocrine cells to insulin-producing cells (overe xpression of $\mathrm{Ngn} 3, \mathrm{Pd} \mathrm{xl}$ and Mafa) [38]. Th is way, somatic cells can be made to induce another differentiated cell type; and these cells have the advantage that they are less prone to tumorigenesis [14].

\section{Heterogeneity of Progenitor Cell Populations in the Stem Cell Niche}

Different studies suggest that great heterogeneity exists in the progenitor cells that make up a stem cell niche. iPSCs and ESCs are very similar when compared at the epigenetic, transcriptional, proteomic and metabolic levels But in detail analyses of human iPSCs and ESCs, they can be distinguished by their expression of protein-coding and non-coding RNAs, chromatin state, residual expression of somatic genes, etc. Many of these differences seem to 
dissipate upon extended induction passing. Reprogramming is not immediately complete upon induction of the endogenous pluripotency network [8]. The fact that residual DNA methylation within lineagespecific genes is found in iPSCs provides tangible evidence that resetting this mark is fundamental to reprogramming, and that failure to do so has a functional consequence. DNA methylation pattern leads to an epigenetic memory of somatic cells. These methylation differences between ESCs and iPSCs are associated with differences at the transcriptional level. Globally, iPSCs are more variable at the molecular level than ESCs.

Pluripotency and self-renewal are the hallmarks of embryonic stem cells. This state is maintained by a network of transcription factors and is influenced by specific signaling pathways. Current evidence indicates that multiple pluripotent states are able to exist in vitro and in vivo [8]. In mammalian systems, the pluripotent cell state exists transiently as the embryo proceeds through the phases of development to give rise to an organism with a diverse array of specialized cell types.

The pluripotent mammalian ESCs and iPSCs states are associated with the in vivo temporal phases of embryo development, and cell in vitro culture time/conditions, respectively. Mouse epiblasts stem cells (mEpiSCs) are derived from the post-implantation epiblast of day 5.5 embryos, in contrast, mouse embryonic stem cells (mESCs) are obtained from epiblast progenitors at day 3.5. Both mEpiSCs and mESCs are similar but not identical. ESCs/iPSCs are referred as "naïve" pluripotent, and EpiSCs as "primed" pluripotent. EpiSCs and ESCs lines grow in different culturing conditions and their transcriptional factors requirement is partially different. Although EpiSCS are termed pluripotent, they have more limited developmental potential than ESCS, and demonstrate heterogeneous expression of early lineagecommitment markers. EpiSCs exhibit reduced expression levels of the transcription factors NANOG, Rex1 and KLF, and exhibit differentiation markers such FGF5 and major histocompatibility complex class I. They also demonstrate heterogeneous expression of early lineage-commitment markers. mESCs and EpiSCs display distinct biological characteristics that are reminiscent of their developmental origin and can be classified into two fundamentally distinct states of pluripotency: 1) the inner cell mass-like or mESCs and 2) the postimplantation epiblast-like state or EpiSCs. In addition, the two states depend on signaling pathways that often antagonize each other. In summary, pluripotent cells isolated in different mammalian species can exist as distinct pluripotent states, and specific extrinsic and intrinsic factors can induce transitions between the states. Reprogramming is still not very efficient from EpiSCs to the ESCs cell state [39].

A growing body of evidence suggests that cell populations, particularly stem cells and progenitor cells in their niches, do not comp rise a ho mogenous cellular entity in vitro or in vivo [37]. Rather, both cells display an inherent heterogeneity at molecular level, which underlies the probabilistic element of their fate determination [40]. Comparison between single human ESCs (hESCs) and single human iPSCs (hiPSCs) lines reveals markedly more heterogeneity in gene expression levels in the hiPSCs, suggesting that hiPSCs occupy an alternate less stable pluripotent state. hiPSCs also displayed slower growth kinetics and impaired directed differentiation compared to hESCs.

hESCs are isolated from explanted blastocysts before implantation and share multiple defining features with mEpiSCs (flat morphology, dependence on FGF2/Activin signaling, etc.) rather than with mESCs. This suggests that hESCs correspond, at least partially, to the primed pluripotent state, and they partially have characteristics of mESCs. It is necessary to use this criterion when we assess the characteristics of the iPSCs.

\section{Initial, Intermediate and Final Steps in the Cell Reprogramming Process from Differentiated Cells to iPSCs Phenotype}

Distinct pluripotent cells are derived during development. Development proceeds from a totipotency state, characteristic of zygote and blastomeres (ESCs). At the 16-cell state, the outer cells of the embryo are allocated to two lineages: the trophoblast lineage and the bipotential inner cell mass, which generates the epiblast and the hypoblast. The epiblast (EpiSCs) will form the embryo (5.5-7.5 days of postimplantation), these cells are termed pluripotent because they are the origin of all somatic and germline cells. Embryonic stem cells (ESCs) first derived from the inner cell mass of blastocyst-stage embryos have the unique capacity of indefinite selfrenewal and potential to differentiate into all somatic cell types. Similar developmental potency can be achieved by reprogramming differentiated somatic cells into induced pluripotent stem cells (iPSCs). Both types of pluripotent stem cells, at different stages of development, provide great potential for fundamental studies of tissue differentiation [41]. ESCs express key pluripotency genes as OCT4, SOX2 and NANOG, they have unlimited proliferation potential, and their genome is highly methylated [42]. ESCs need LIF and BMP4 for growth in in vitro cultivation, and support stabilization mESCs by inducing inhibitors of differentiation $(I d)$ genes. A core set of transcription factors (OCT4, NANOG, SOX2 and Tcf3) maintains the pluripotent state of ESCs.

The low efficiency of the reprogramming process to generate iPSCs with OSKM has been analyzed and suggests that the process must encounter epigenetic barriers that can be seen as roadblocks in the journey to pluripotency. Furthermore, many different cell types are reprogrammed with similar kinetics under almost identical reprogramming conditions, but the kinetics and efficiency of the process can dramatically differ amongst cell types. Thus, less differentiated cells (progenitors) could be reprogrammed more efficiently than differentiated cells because the progenitor transcriptome is less different to that of the ESCs [13].

As we have discussed, ESCs and iPSCs are equivalent but not identical. ESCs derived from pre-implantation blastocysts and iPSCs are like to ESCs derived from postimplantation it. Total gene expression comparison between ESCs and iPSCs revealed some differences between the two in pluripotency transcription factors, protein-coding genes, miRNA expression profiles, large intergenic non-coding RNAs, epigenetic changes, (pattern 
of DNA methylation and post-translational histone modifications to the ES cell-like state) etc. [43].

To improve transcription factor-induced reprogramming efficiency (and translation applications of iPSCs), it is crucial to understand the temporal order of molecular events leading to pluripotency. Recent live imaging of mouse fibroblast undergoing reprogramming supports the idea that reprogramming proceeds through highly synchronized progressive events, with first events initiating almost immediately after induction of the "master" reprogramming factors. Even though reprogramming events are initiated early, the presence of reprogramming factors is required until the end, but most cells in which these initial reprogramming events occur do not complete reprogramming. Briefly, the reprogramming process begins by the induction of proliferation and downregulation of fibroblast specific transcription is followed by the acquisition of epithelial characteristics and activation of some markers. Later, pluripotency related genes are activated. Markers used to detect these steps include alkaline phosphatase and stage-specific embryonic antigen 1 (SSEA1) at an intermediate stage; and NANOG (mouse system) or the surface marker TRA$1-60$ in human cells for final stages $[8,44]$.

The first characterized initiation event during reprogramming of fibroblast is the increase of cell cycle rate, from 22 to 12 hours cell cycle length (after 1 - 2 days of induction with the reprogramming factors). The cell cycle seems to have an important role in reprogramming, and great reductions in latency can be achieved by increasing cell proliferation, and a concomitant decrease in cell size. These changes occur along with the induction of proliferation genes and downregulation of the somatic expression programme [22,27]. Only a minority of the fibroblasts starts to divide faster, and the majority of the OSKM-overexpressing fibroblasts retains their slowdividing nature and fails to reprogram, and often undergo apoptosis and senescence [12]. Specifically, the silencing of central regulators or tumor suppressor genes of these responses, such as p53 and p21 or p16/INK4A (also known as CDKN2A) is observed upon reprogramming, and their experimental depletion enhances the efficiency and kinetics of iPSCs generation.

ESCs and iPSCs have characteristics of epithelial cells, with tight intracellular contacts and surface expression of the key epithelial cadherin (E-cadherin). Thus, mesenchymal cells such as fibroblasts need to gain an epithelial character during reprogramming. After suppression of the somatic transcriptional programme, small and fast-cycling cells, undergo coordinated changes in cell-cell and cell-matrix interactions, which correspond with a loss of mesenchymal features and acquisition of epithelial cell characteristics. At 4-8 days later, some of the small cycling cells form compact colonies, concurrent with a mesenchymal-to-epithelial transition (MET). MET is a reversible biological process involving the transition from motile, multipolar or spindle-shaped mesenchymal cells to planar arrays of polarized epithelial cells; MET is exactly the opposite of the epithelial-mesenchymal transition. Signaling pathways that are known to promote or suppress MET, affect the efficiency of the reprogramming process. Like this, the inhibition of signaling by transforming growth factor- $\beta$ improves reprogramming. In contrast, bone morphogenetic protein signaling enhances reprogramming [28,29].

In late events toward pluripotency of a fibroblast after MET, the cells erase the epigenetic memory, gain naïve pluripotency capabilities, upregulate the pluripotency genes, reactive the chromosome X (in females), keep ESSlike transcripto me-epigenome-DNA replication-cell cycle, and acquire global genome reorganization based on characterization of replication timing (Figure 2) [7,22]. OCT4 and SOX2 suppress the pro-mesenchymal regulator SNAIL1, whereas KLF4 induces the epithelial programme by directly binding and activating epithelial genes, including E-cadherin; at the same time, MYC reduces TGF $\beta$ signaling.

As we referred above, many fibroblasts in OSKMexposed conditions are not driven to generate iPSCs colonies and are transformed in partially reprogrammed cells. Some of them show ES cell-morphology, but do not express endogenous pluripotency factors like NANOG or Esrrb, and start to appear in the reprogramming culture after 4-7 days post induction of reprogramming factors. In experimental attempts to clonally expand these colonies, some of them regress or apoptose, but a few can be maintained as stable lines and are referred to as to partially reprogrammed cells or pre-iPSCs. Typically, the somatic transcriptome is efficiently downregulated in pre-iPSCs, but most of the key pluripotency genes are not upregulated $[12,22]$. Pre-iPSCs have shown to retain fibroblast-like DNA-hypermethylated regions, most notably the NANOG and OCT4 loci, which are typically robustly demethylated in iPSCs/ESCs. Other studies have shown that the missing cofactors and the repressive chromatin state are a barrier to reprogramming factors binding in pre-iPSCs [14]. A correlation of the genome-wide locations of reprogramming factors at the pre-iPSC stage, with the absence or presence of a wide range of chromatin marks and nucleosomal positioning, will further our understanding of how the reprogramming factors engage chromatin at key pluripotency genes [14,24]. In particular, stable partially reprogrammed cells only reactivate a subset of stem cell-associated genes and fail to repress many genes associated with differentiation. Although these cells have not managed to attain pluripotency, additional manipulation can coax them into completing this last step.

In the final stage of reprogramming, the cells attain and maintain pluripotency, switching to the endogenous programme and, in so doing, shutting down the exogenous transgenes. At this stage, NANOG becomes a crucial determinant. Finally, the endogenous pluripotency programme needs to be activated for the cell to become independent from the exogenous transgenes. This occurs late in reprogramming and may involve subduing repressive mechanisms such as histone and DNA methylation. Once the endogenous programme is in place, the self-regulatory mechanisms that are associated with OCT4, SOX2, and NANOG help to maintain pluripotency. Importantly, there must be a complete repression of lineage specifying genes, therefore, the genes that promote differentiation are inactive. This is achieved through the regulation of DNA methtyltransferases by OCT4, SOX2 and NANOG, which play a crucial part in repressing lineage-specifying genes and silence exogenous transgenes [45]. 
The regulatory regions of some pluripotency related genes, such as OCT4, SOX2, undifferentiated embryonic cell transcription factors (UTFs), developmental pluripotency associated 5 (DPPPA5), zinc finger protein 42 (ZFP42), and DPPPA3 are hypermethylated at the DNA level in somatic cells and pre-iPSCs, and lack the activation mark histone $\mathrm{H} 3$ lysine 4 trimethylation (H3K4me3). DNA demethylation and loss of repressive histone methylation marks in the pluripotenty genes probably occur at the end of the reprogramming process, concomitant with the binding of the reprogramming factors OCT4, SOX2 and KLF4 and the transcriptional upregulation of these genes. Chromatin states influence reprogramming at various stages. It has been found that in the early phase of reprogramming, transcriptional changes are limited to those promoters that carry histone $\mathrm{H} 3 \mathrm{~K} 4 \mathrm{me} 3$; a histone modification that is strongly associated with transcriptional activation. During

$\begin{array}{ll}\text {-Lineage factors } & \text {-Expression of } \\ \text { expressed } & \text { chromatin remodeling } \\ \text {-Peripheral } & \text { factors } \\ \text { heterochromatin } & \text {-Changes in chromatin } \\ \text {-Extended G1 phase } & \text { state and in DNA } \\ \text {-Specialized cell } & \text { methylation } \\ \text { morphology } & \text {-Repression of } \\ \text {-DNA methylation at } & \text { developmental genes } \\ \text { pluripotency promoters } & \text {-Re-entry into cell cycle } \\ & \text {-Mesenchymal- } \\ & \text { epithelial transition }\end{array}$

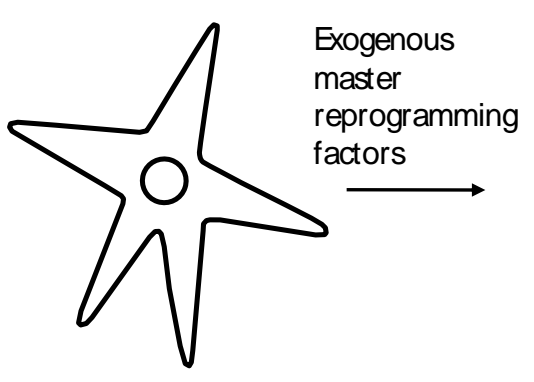

Terminally differentiated cell
Initial reprogrammed reprogramming, silencing of somatic genes is associated with a change in chromatin structure at their enhancers and promoters, and in particular with a rapid loss of his tone H3K4me3 [46].

Taken together, the identification of these events indicates that a particular, albeit ill-defined, sequence of stages must be traversed to attain pluripotency. The main phases, together with characteristics and events in differentiation and reprogramming in mouse systems, are shown in Figure 2. Dedifferentiation and reprogramming seem outwardly very similar, as they both can induce a differentiated cell to regress. During reprogramming, an initial still unknown event occurs that allows the exogenous factors to induce pluripotency, and it probably depends on the cell heterogeneity of the terminally or partially differentiated niche population (transcriptional and epigenetic profiling).

-Gain of naive
pluripotency
capabilities
-Upregulation of
pluripotency genes
-ES-like
transcriptome/epigen
ome DNA replication-
cell cycle
-Erasure of
epigenetic memory

-Unlimited self-renewal
-Hyperdynamic
chromatin
-Short G1 cell cycle
-High level of
pluripotency regulators
High nuclear to
cytoplasmic ratio
-no DNA methylation at
pluripotency promoters
-Endogenous
expression of pluripotent
genes

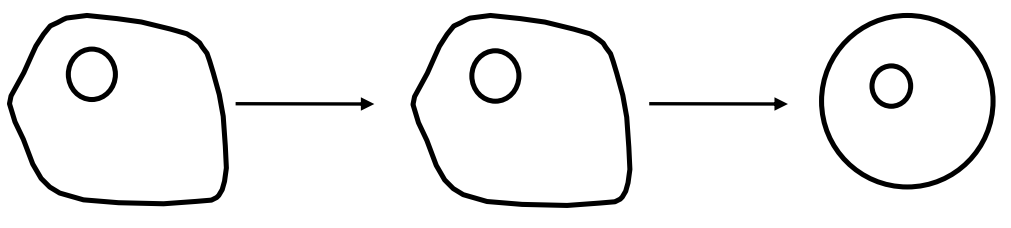

Induced proliferate stem cell

Figure 2. Main phases and subphenotype changes in the somatic cellular reprogramming process [13]

\section{Dynamics and Cellular Heterogeneity in the Differentiation/Dedifferentiation Process}

Stem cells must retain their ability of self-renewal in order to maintain tissue homeostasis. They need to exit this self-renewal cycle and instead proliferate and differentiate when instructed by mic roenvironmental cues. The balance between self-renewal and proliferation and terminal differentiation is strictly regulated.

As we have mentioned, the reprogramming efficiency to generate iPSCs appears to be quite low. Determination of reprogramming-efficiency and kinetics of in vitro reprogramming is typically based upon the appearance of iPSCs colonies (typically 2-6 weeks). Efficiency is calculated by the fraction of reprogrammed cells (pluripotency markers) divided by the total number of plated cells. A large number of conditions of in vitro culture are not considered in the ultimate results. Several lines of evidence suggest that an important rate-limiting epigenetic event for reprogramming may be the reactivation of the key exo/endogenous, and by autoregulatory circuitry that maintain the ESC state [41]. It is still partially known how many of these epigenetic changes are rate limiting for reprogramming.

Different parameters may affect gene expression and biological characteristics of ESCs/iPSCs as transgenecontaining iPSCs, genetic background, incomplete reprogramming, in vitro molecular heterogeneity among ESCs/iPSCs, reprogramming factor combinations and routes, and progressive state of the pluripotent stem cells. Reprogramming of somatic cells into iPSCs requires the resetting of the epigenetic state from a somatic to a pluripotent embryonic state. Nuclear transfer might reset the epigenetic characteristics of somatic cells more effectively than reprogramming in vitro with transcription factors [47]. The pluripotent epigenetic state may represent a default cellular state, and iPSCs may have a growth advantage over somatic cells. 
The control of somatic cell reprogramming integrates signaling pathways and molecular mechanisms [7,11]. These intracellular signaling pathways can be activated or inhibited in order to improve the efficiency of somatic cell reprogramming. For instance, $\mathrm{Wnt} / \beta$-catenin is known to have an important role in the maintenance of ESC selfrenewal. The activation of this signaling or the phosphoinositide 3-kinase PI3/Akt, or the inhibition of glycogen synthase kinase (GSK) $3 \beta$, strongly improve the reprogramming process. In contrast, the pathways that normally stimulate ESCs differentiation are usually inhibited for the enhancement of reprogramming efficiency. For example, inhibition of the MEKMAPK/ERK pathway that normally influences ESC differentiation, promotes completion of the reprogramming processes [7]. Putative interconnections between players of different signaling pathways are partially known. The kinetics of these processes involves a large number of intracellular signaling events that modulate the frequency and completion of the mechanisms that forms a fully reprogrammed clone. Concomitant with expression of exogenous OCT4, SOX2 and NANOG, fibroblasts start to repress developmental genes and induce several repressive epigenetic enzymes (such as NuRD and Setdb1 complexes) that configurate chromatin-remodelling factors (Smarcad1, Myst3, Jmjd2c and Jmjd 1a) and in re-entry in to the cell cycle (e.g. p53 inhibition, specific miRNAs). Core transcription factors in ESCs co-regulate expression of epigenetic factors that participate in maintenance of self-renewal and pluripotency. Changes in the global chromatin state that are necessary for full reprogramming can also arise from recruitment of the repressive Polycomb proteins [48]. Once this first barrier has been overcome, the cells can attain intermediate states, in which they are pre-iPSCs, where the expression of endogenous genes of pluripotency is not completely reactivated. Only after complete chromatin remodeling occurs, the expression of endogenous pluripotent genes (OCT4, SOX2, KLF4, cMYC, etc) is re-activated, at the same time the complex protein network that controls pluripotency even after silencing of ectopic OCT4 and SOX2. In these second step proliferation, antiapoptotic, and adhesion/motiling signaling networks are positively activated (Figure 2).

\section{Concluding Remarks}

Although rapid progress in our understanding of cellular differentiation process has been made, a number of important questions remain unanswered. Reprogramming somatic cells to a pluripotent state was used as model to identify the dynamic changes of genetic/epigenetic and proteomic participant factors in the cellu lar differentiation process.

The differentiated cellular state is not irreversible. Changes in gene expression by genetic/epigenetic patterns may not only restore pluripotency (dedifferentiation) in a fully differentiated cell state, but may also induce the cell in differentiation, redifferentiation and transdifferentiation processes.

Current direct reprogramming approaches are inefficient and involve stochastic changes occurring in highly heterogeneous cell populations in a niche tissue.
Because only a small fraction of somatic cells generate iPSCs (versus pre-iPSCs) in culture conditions, the information gained from molecular analyses of the heterogeneous intermediate cell populations is limited. Such analyses cannot distinguish between the rate-limiting and non-rate-limiting epigenetic changes in those cells in reprogramming assays.

\section{Statement of Competing Interests}

The authors have no competing interests.

\section{List of Abbreviations}

iPSCs: $\quad$ induced pluripotent stem cells .

OSKM: four OCT4, SOX2, KLF4 and c-MYC

factors that are able to restore pluripotency.

ES Cs: embryonic stem cells.

LIF: cytokine leukaemia inhibitory factor.

BMP4: $\quad$ bone morphogenic protein 4.

hESCs: human embryonic stem cells.

CNVs: copy number variants.

DMRs: differentially methylated regions in

genome.

EMT: epithelial-to mesenchymal transition.

SNAIL1: transcription factors snail homolog, repressor of E-cadherin. Their exp ression induces EMT.

Wnt: Signaling pathways that controls cellcell-communication in the embryo and adult. In Wnt signaling pathways participate the wingless or Wnt gene family.

JAK: Janus tyrosine kinase.

mEpiSC: mouse epiblasts stem cells derived from the post-implantation epiblast of day 5.5 e mbryos.

mESCs: mouse embryonic stem cells obtained from epiblast progenitors at day 3.5.

hiPSCs: human induced pluripotent stem cells.

SSEA: $\quad$ stage-specific e mbryonic antigen 1.

MET: mesenchymal-to-epithelial transition

UTFs: undifferentiated embryonic cell

transcription factors.

DPPPA5: developmental plu ripotency associated 5 factor.

ZFP42: $\quad$ zinc finger protein 42 .

GS K3: $\quad$ glycogen synthase kinase 3.

\section{References}

[1] Iglesias-Bartolome R, Gutkind JS.” Signaling circuitries controlling stem cell fate: to be or not to be". Current Opin Cell Biol, 23(6):716-723, Dec. 2011

[2] Cohen DE, Melton D. "T urning Straw into gold: directing cell fate for regenerative medicine". Nat Rev Gen,12 (4):243-252. Apr. 2011

[3] Izpisua JCB. "Reprogramming development and aging: cell differentiation as a malleable process". Curr Opin Cell Biol, 24:713-715. Dec. 2012.

[4] Sabour D, Schoeler HR. "Reprogramming and the mammalian germline: the Weismann barrier revisited". Curr Opin Cell Biol, 24:716-723. Dec. 2012.

[5] Takahashi K, Yamanaka S. "Induction of pluripotent stem cells from mouse embryonic and adult fibroblast cultures by defined factors". Cell,126 (4):663-676. Aug. 2006. 
[6] Takahashi K, Tanabe K, Ohnuki M, Narita M, Ichisaka T, Tomoda $\mathrm{K}$, Yamanaka S. "Induction of pluripotent stem cells from adult human fibroblast by defined factors". Cell, 131:861-872. Nov. 2007.

[7] Sanges D. Cosma MP." Reprogramming cell fate to pluripotency: the decision-making signaling pathways". Int J Dev Biol, 54 (1112):1575-1587. 2010.

[8] Plath K, Lowry WE. "Progress in undestanding reprogramming to the induced pluripotent state". Nat Rev Gen, 12(4):253-26., Apr. 2011.

[9] Nie B, Wang H, Laurent T, Ding S. "Cellular reprogramming: a small molecule perspective". Curr Opin Cell Biol, 24:784-792. Dec. 2012.

[10] Narsinh KH, Sun N, Sanchez-Freire V, Lee AS, Almeida P, Hu S, et al. "Single cell transcriptional profiling reveals heterogeneity of human induced pluripotent stem cells". J Clin Invest,12 1(3):12171222. Mar. 2011.

[11] Valdespino V, Valdespino PM, Valdespino VJr. "Genomicepigenomic signaling pathways changes in celular differentitation process". Am J Biomed Res, 1(2):35-42, 2013.

[12] Stadtfeld M, Maherali N, Breaullt DT, Hochedlinger K. "Defining molecular comerstones during fibroblast to iPS cell reprogramming in mouse". Cell Stem Cell, 2(3):230-240. Mar. 2008.

[13] Paap B, Plath K. "Reprogramming to pluripotency: stepwise resetting of the epigenetic landscape". Cell Res, 21(3):486-501. Mar. 2011

[14] Taby R. Ossa JP. "Cancer epigenetics". CA Cancer J Clin, 60(6):376-392.Nov-Dec. 2010.

[15] Polo JM, Liu S, Figueroa ME, Kulalert W, Eminli S, Tan KY, et al. "Cell type of origin influences the molecular and functional properties of mouse induced pluripotent stem cells". Nat Biotechol, 28(8):848-855. Aug. 2010.

[16] Rando TA, Chang HY. "Aging, rejuvenation, and epigenetic reprogramming: resetting the aging clock". Cell, 148 (1-2):46-57. Jan. 2012.

[17] Mahmoudi S, Brunet A. "Aging and reprogramming: a tow-way street”. Curr Opin Cell Biol, 24:744-756. Dec. 2012.

[18] Freije JMP, Lopez-Otin C. "Reprogramming aging and progeria". Curr Opin Cell Biol, 24:757-764. Dec. 2012.

[19] Liu GH, Ding Z, Izpisua JCB. "IPSC technology to study human aging and aging-related disorders". Curr Opin Cell Biol, 24:765774. Dec. 2012.

[20] Furusawa C, Kaneko K. "A dynamical-systems view of stem cell biology”. Science, 228(6104):215-217. Oct. 2012.

[21] Xu RH, Peck RM, Li DS, Feng X, Ludwing T, Thomson JA. "Basic FGF and suppression of BMP signaling sustain undifferentiated proliferation of human ES cells". Nat Methods, 2 (3):185-190. Mar. 2005.

[22] Ng HH, Surani MA. "The transcriptional and signaling networks of pluripotency". Nat Cell Biol, 13(5):490-496, May.2011.

[23] Banito A, Rashid ST, Acosta JC, Li S, Pereira CF, Geti I, et al. "Senescence impairs successful reprogramming to pluripotent stem cells". Genes Dev, 23(18):2134-2139. Sep. 2009.

[24] Meshorer E, Misteli T. "Chromat in in pluripotent embryonic stem cells and differentiation". Nat Rev Mol Cell Biol, 7(7):540-546. Jul. 2006.

[25] Heng JC, Feng B, Han J, Jiang J, Kraus P, Ng JH, et al. "The nuclear receptor $\mathrm{Nr} 5 \mathrm{a} 2$ can replace Oct4 the reprogramming of murine somatic cells to pluripotent cells". Cell Stem Cell, 6(2):167-174. Feb. 2010.

[26] Denholtz M, Plath K." Pluripotency in 3D:genome organization in pluripotent". Cell Stem Cell, 24(6):793-801. Dec. 2012.

[27] Zhu S, Li W, Zhou H, Wei W, Ambasudhan R, Lin T, et al. "Reprogramming of human primary somatic cells by OCT4 and chemical compounds" Cell Stem Cell, 7(6):651-655. Dec. 2010.
[28] Samavarchi-Tehrani P, Golipour A, David L, Sung HK, Beyer TA Datti A, et al. "Functional genomics reveals a BMP-driven mesenchymal-to-epithelial transition in the initiation of somatic cell reprogramming" Cell Stem Cell, 7(1):64-77. Jul. 2010.

[29] Li R, Liang J, Ni S, Zhou T, Qing X, Li H, He W, et al. "A mesenchymal-to-epithelial transition initiates and is required for the nuclear reprogramming of mouse fibroblast". Cell Stem Cell, 7(1):51-63. Jul. 2010.

[30] Castanon I, Gonazlez-Gaitan M. "Oriented cell division in vertebrate embryogenesis” Curr Opin Cell Biol, 23:697-704. Dec. 2011

[31] Hussein SM, Batada NN, Vuoristo S, Ching RW, Autio R, Narva E, et al. "Copy number variation and selection during reprogramming to pluripotency". Nature, 471(7336): 58-62. Mar. 2011.

[32] Lister R, Pelizzola M, Kida YS, Hawkins RD, Nery JR, Hon G, et al. "Hotspots of aberrant epigenomic reprogramming in human induced pluripotent stem cells". Nature, 471(7336):68-73. Mar. 2011.

[33] Robinton DA, Daley GQ. "The promise of induced pluripotent stem cells in research and therapy". Nature, 481(7381):295-306. Jan. 2012.

[34] Maeda Y." Cell-cycle checkpoint for transition from cell division to differentiation". Develop Growth Differ, 53:463-481. 2011.

[35] Katto J, Mahlknecht U. "Epigenet ic regulation of cellular adhesion in cancer". Carcinogenesis, 32(10):1414-1418. Jun. 2011.

[36] Klusza S, Deng WM. "At the crossroads of differentiation and proliferation: precise control of cell-cycle changes by multiples signaling pathways in Drosophila follicle cells". Bioessays, 33(2):124-134. Feb. 2011

[37] Poulson ND, Lechler T. "Asymmetric cell divisions in the epidermis". Int Rev Cell Mol Biol, 295:199-232. 2012.

[38] Holmberg J and Perlmann. "Maintaining differentiated cellular ident ity”. Nat Rev Genet, 13 (6): 429-439. June 2012.

[39] Enver T, Pera M, Peterson C, Andrew PW. "Stem cell states, fates and the rules of attraction”. Cell Stem Cell, 4(5):387-397. May. 2009

[40] Guo G, Huss M, Tong GQ, Wang C, Li Sun L, Clarke ND, Robson P. "Resolution of cell fate decisions revealed by single cell gene expression analysis from zy gote or blastocyst". Dev Cell, 18(4):675-685. Apr. 2010.

[41] Hanna JH, Saha K, Jaenisch R.” Pluripotency and cellular reprogramming: facts, hyphoteses, unresolved issues". Cell, 143 (4):508-524, Nov. 2010.

[42] Li M, Liu GH, Izpisua BJC. "Navigat ing the epigenetic landscape of pluripotent stem cells". Nat Rev Mol Cell Biol, 13(8):524-535, Aug. 2012.

[43] Chin MH, Mason MJ, Xie W, Volinia S, Singer M, Peterson C, et al. "Induced pluripotent stem cells and embryonic stem cells are distinguished by gene expression signatures". Cell Stem Cell, 5(1):111-123. Jul. 2009.

[44] Winkler T, Cantilena A, Metais JY, Xu X, Nguyen AD, Borate B, et al. "No evidence for clonal selection due to lentiviral integration sites in human induced pluripotent stem cells". Stem Cells, 28(4):687-694. Apr. 2010.

[45] Jopling C, Boue S, Izpisua JCB." Dedifferentiation, transdifferentiation and reprogramming: three routes to regeneration". Nat Rev Mol Cell Biol, 12(2):79-89. Feb. 2011.

[46] Loh YH, Yang L, Yang JC, Li H, Collins JJ, Daley GQ."Genomic approaches to deconstruct pluripotency". Annu Rev Genomics Hum Genet, 12:165-185. Sep.2011.

[47] Tachibana M, Amato P, Sparman M, Marti NG, Tippner-Hedges R, Ma H, Kang E, et al. "Human embryonic stem cells derived by somatic cell nuclear transfer". Cell, 153:1-11. May. 2013.

[48] Boyer LA, Plath K, Zeitlinger J, Brambrink T, Medeiros LA, Lee TI, et al. "Polycomb complexes repress developmental regulators in murine embryonic stem cells". Nature, 441 (7091):349-353. May. 2006. 